\title{
Educational status is related to mortality at the community level in three areas of Tanzania, 1992-1998
}

Philip Setel, David Whiting, Yusuf Hemed, K G M M Alberti, for the Adult Morbidity and Mortality Team, Tanzanian Ministry of Health

Research on health outcomes and socioeconomic status in Africa remains scanty. ${ }^{1}$ There continues to be a dearth of representative mortality data from developing countries, particularly from sub-Saharan Africa. This lack of information hampers evidence-based planning and an understanding of the relation between relative socioeconomic advantage and health outcomes such as mortality. In the United States, Murray et al have demonstrated the utility of examining mortality data from small geographical areas in relation to socioeoconomic status represented by variables such as ethnicity and education. Here we present data from a large demographic surveillance system in the United Republic of Tanzania demonstrating a strong relation between average years of formal education among adults (as an indicator of social position) and mortality. We use formal education in the adult population as an indicator of relative socioeconomic advantage in 29 small geographical units from three areas in Tanzania.

Participants, methods, and results

Since 1992, the Adult Morbidity and Mortality Project (AMMP) of the Tanzanian Ministry of Health in partnership with the University of Newcastle upon Tyne has collected community-based mortality data through a demographic surveillance system. The total population covered is over 330000 in three areas of Tanzania including a poor rural district, a relatively well off rural district, and poor and middle income urban areas. We use annual and semi-annual censuses to determine population size and characteristics. Continuous mortality reporting based on verbal necropsy interviews provides all cause and cause specific mortality for all ages. Details of methods, including cause of death attribution using verbal necropsy, have been published previously. ${ }^{3}$ Population denominators are the total resident population at the midpoint of the period of analysis, and mortality was determined from all incident deaths at all ages in the resident populations from July 1992 to June 1998. We have analysed data at the level of "wards" and "branches." A ward is a geographically defined area made up of several contiguous rural villages; a branch is made up of contiguous urban neighbourhoods. The average size of wards (average population 11337 ) and branches (average population 9251) are comparable.

Figure 1 shows the relation $\left(r^{2}=0.507\right)$ between all cause mortality rates at the ward/branch level, and education in the adult population aged 15-49. Further analysis also revealed an association between maternal mortality ratio at ward/branch level and adult female education $\left(r^{2}=0.404\right)$. A greater number of years of completed education among all adults correlated strongly with lower levels of under 5 mortality $\left(r^{2}=0.560\right)$.

\section{Comment}

In the AMMP areas, higher educational status at the community level relates strongly to lower total and maternal mortality. In Africa south of the Sahara this relation has, to our knowledge, only been explored in relation to child and maternal mortality. ${ }^{4}$ Differences in adult educational attainment account for $51 \%$ of the observed difference in all cause mortality and $40 \%$ of the difference in maternal mortality, and $56 \%$ of the difference in under 5 mortality. Although it has been postulated that an inverse relation exists between educational level of women and levels of maternal and perinatal mortality, we emphasise that this is an association and not necessarily a direct cause

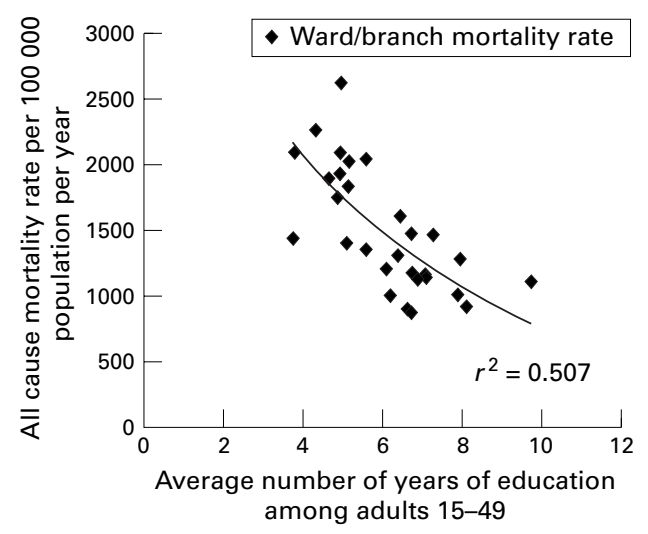

Figure 1 All cause mortality rate by average number of years of education in 29 wards in Tanzania. 
and effect relation, as mediating factors may exist. Educational level may reflect earning capacity and economic status as well as health related behaviours and attitudes. It is also possible that poorer villages with lower educational attainment may have different access to and patterns of using health facilities. ${ }^{4}$ The correlates between education and mortality in these Tanzanian data are striking and strongly support the need for further investigation into the role of education as an essential component of health improvement in developing countries.

Funding: This publication is an output of a project of the Tanzanian Ministry of Health funded by the UK Department for International Development (DFID) and implemented in partnership with the University of Newcastle upon Tyne. The views expressed are not necessarily those of DFID.
Conflicts of interest: none.

1 Morris SS, Carletto C, Hoddinott J, et al. Validity of rapid estimates of household wealth and income for health surveys in rural Africa. $\mathfrak{J}$ Epidemiol Community Health 2000;54:381-7.

2 Murray CJL, Michaud CM, McKenna MT, et al. U.S Patterns of mortality by county and race: 1965-1994. Cambridge, AL: Harvard Burden of Disease Unit, Harvard Center for Population and Development Studies/US Centers for Disease Control and Prevention, 1998.

3 Kitange HM, Machibya H, Black J, et al. Outlook for survivors of childhood in sub-Saharan Africa: adult mortality in Tanzania. BMF 1996;312;216-20.

4 Mbacké C, van de Walle E. Socio-economic factors and use of health services as determinants of child mortality. In: van de Walle E, Pison G, Sala-Diakanda M, eds. Mortality and society in sub-Saharan Africa. Oxford: Clarendon Press, 1992:123-44.

5 Urassa E, Lindmark G, Nystrom L. Maternal mortality in Dar es Salaam, Tanzania: socio-economic, obstetric history and accessability of health care factors. American fournal of Health Sciences 1995;2:242-9. 